\title{
Laser power noise detection at the quantum-noise limit of 32 A photocurrent
}

\author{
Patrick Kwee, * Benno Willke, and Karsten Danzmann \\ Max-Planck-Institut für Gravitationsphysik (Albert-Einstein-Institut) and Leibniz Universität Hannover, \\ 30167 Hannover, Germany \\ *Corresponding author: patrick.kwee@aei.mpg.de
}

Received July 15, 2011; accepted August 8, 2011;

posted August 18, 2011 (Doc. ID 151204); published September 8, 2011

\begin{abstract}
The power noise of a cw Nd:YAG laser system was measured at radio frequencies using the optical ac coupling technique. An additional mode cleaner in the setup allowed a high optical ac coupling amplification of 62.3. For the first time, to our knowledge, a sensitivity of $1.1 \times 10^{-10} \mathrm{~Hz}^{-1 / 2}$ relative power noise was achieved corresponding to an equivalent detected photocurrent of $32 \mathrm{~A}$. High precision optics experiments can utilize this scheme to improve the sensitivity of their photodetectors. (c) 2011 Optical Society of America
\end{abstract}

OCIS codes: $040.5160,230.5750$.

Sensitive laser power detection is essential in many high precision optics experiments to convert optical signals to electrical ones that can be acquired and processed. Quantum noise is the fundamental sensitivity limit in the detection. The linear spectral density of the relative power noise due to quantum noise is proportional to $1 / \sqrt{P}$, where $P$ is the detected laser power. Thus, a high laser power needs to be detected to achieve a high signalto-quantum-noise ratio. To reach, e.g., a relative power noise level of $10^{-10} \mathrm{~Hz}^{-1 / 2}$, a power of at least $37 \mathrm{~W}$ has to be detected [1]. The high laser power causes many technical problems in the photodetectors used today, such as photodiode saturation, dynamic range limits of the readout electronics, or thermal effects in the photodiode.

A different approach is the optical ac coupling technique [2,3]. It allows to significantly improve the sensitivity of a photodetector without increasing the average power on the photodetector. In this scheme, it is the power fluctuations in reflection of a nearly impedance-matched optical resonator that are directly detected.

In this Letter, we report an improved optical ac coupling scheme used to measure the laser power noise at radio frequencies of a $\mathrm{cw}$ solid-state laser system at $1064 \mathrm{~nm}$ wavelength. We achieved a quantum-noiselimited sensitivity of $1.1 \times 10^{-10} \mathrm{~Hz}^{-1 / 2}$ relative power noise at $27 \mathrm{MHz}$. To our knowledge, this sensitivity surpassed the best sensitivity reported so far [2] by almost 1 order of magnitude. Furthermore, our results showed that this laser system had no unexpected power noise in the frequency range relevant for hetrodyne detection schemes.

The key element in this sensitivity improvement was an additional linear resonator placed between the resonator used for the optical ac coupling (called ac coupling resonator, ACC) and the photodetector. The optical ac coupling technique is prone to be limited by parasitic modes, such as higher-order transversal modes, and therefore typically requires laser beams of extremely high beam quality. Although our laser system had 99.5\% of its power in the $\mathrm{TEM}_{00}$ mode, the remaining higherorder TE magnetic (TEM) modes would limit the optical ac coupling amplification to $g<8$ [3]]. The additional resonator efficiently suppressed these parasitic modes, achieving a much higher amplification of up to $g=62.3$.

This experiment is relevant, e.g., for current and next generation ground-based interferometric gravitational wave detectors [4], which require high sensitivity power detection for laser stabilization $[5,6]$. We demonstrated the high sensitivity, the high-power compatibility, and a topology that can be employed in gravitational wave detectors.

In our experimental setup (Fig. 1) we used a cw prestabilized laser system [5,7] with an output power of about $130 \mathrm{~W}$ at a wavelength of $1064 \mathrm{~nm}$. The power

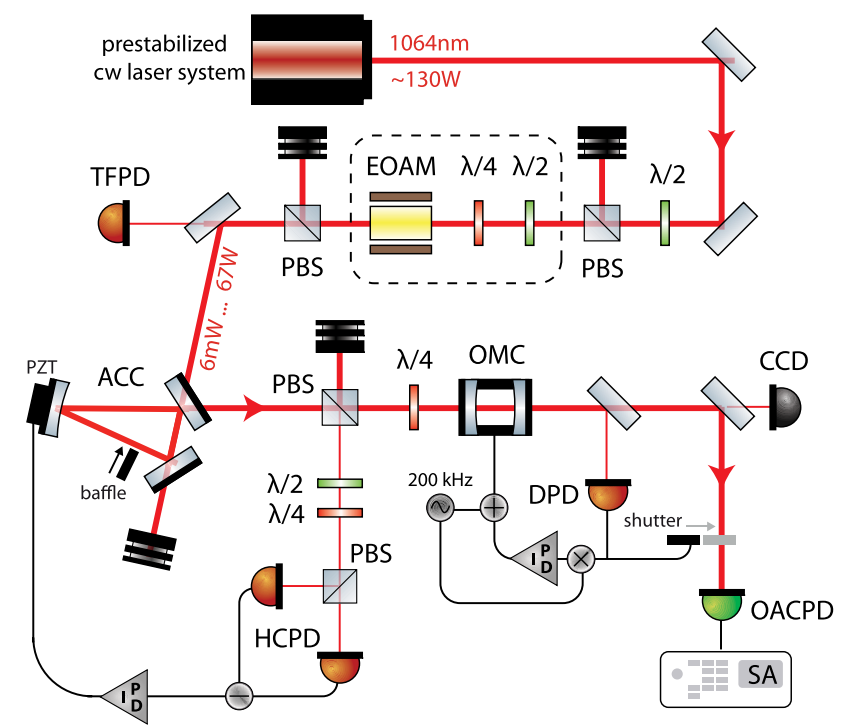

Fig. 1. (Color online) Experimental setup: The beam of the laser system is coupled into the resonator ACC. The power noise was measured with the photodetector OACPD. The mode cleaner OMC is used to suppress parasitic higher TEM modes that would otherwise limit the sensitivity of the power detection. The components in the dashed box were used for calibration and were placed in the beam path only at low power $(\approx 2 \mathrm{~W})$. Reference measurements were performed with photodetector TFPD. DPD, HCPD, photodetectors; SA, spectrum analyzer; PBS, polarizing beam splitter; $\lambda / 2, \lambda / 4$, wave plates. 
for the downstream experiment was adjusted with a $\lambda / 2$ wave plate and a subsequent polarizing beam splitter. An electro-optic amplitude modulator (EOAM) was used to create amplitude modulations for calibration purposes. Since this modulator was not high power compatible it was placed in the beam only for low power experiments $(\approx 2 \mathrm{~W})$.

A ring resonator with a design similar to the one in [2] was used as an ACC. The finesse was about 50 and the bandwidth $7 \mathrm{MHz}$. The ACC was stabilized to a fundamental mode resonance using the Hänsch-Couillaud sensing scheme [8] via photodetector HCPD, a piezoelectric element $(\mathrm{PZT})$ to change the resonator round trip length, and a feedback control loop with a bandwidth of about $10 \mathrm{kHz}$. The impedance matching [9], critical for the optical ac coupling technique, was adjusted by two methods: by tilting one mirror of the resonator, the fundamental mode incidence angles on the mirrors were changed. Because of an angle dependence of the mirror transmission, the impedance matching was adjusted to be slightly overcoupled. The second method used a micrometer screw as a resonator internal baffle to create additional resonator losses. Thus, we were able to easily choose any impedance matching from overcoupled, to impedance matched, to undercoupled.

The beam reflected by the ACC was transmitted through the linear resonator optical mode cleaner (OMC). This resonator was used as mode cleaner [10] to suppress higher-order TEM modes (parasitic modes) that would cause additional power noise at the optical ac-coupled detector (OACPD) and would limit the sensitivity of the experiment. Two mirrors were glued to a PZT acting as spacer. This resonator had a free spectral range of $19.2 \mathrm{GHz}$ and a bandwidth of $104 \mathrm{MHz}$. We used a dither lock scheme with a modulation frequency of $200 \mathrm{kHz}$ to stabilize the resonator to a fundamental mode. We verified with a CCD camera in transmission that the resonant mode was the fundamental mode. The signal of the photodetector DPD was demodulated to generate error signals. A feedback control loop with $12 \mathrm{kHz}$ bandwidth controlled the resonator length. The signal of this photodetector was also used to trigger an interlock to protect the photodetector OACPD from excessive laser power in case the ACC became nonresonant. We used a two-staged shutter system with $6 \mu \mathrm{s}$ and $20 \mathrm{~ms}$ reaction time.

The alignment to the linear resonator OMC was critical to achieve a high throughput of the fundamental mode and a high suppression of parasitic modes. We used an alignment beam (not shown in Fig. 1) coupled to the countercirculating fundamental mode of the ACC to align the beam to the OMC eigenmode.

The best sensitivity for power noise was above the bandwidth of the ACC and below the bandwidth of the OMC. The calculated sensitivity peak was at $27 \mathrm{MHz}$.

We used two different types of photodetectors as OACPD for different power levels. For measurements up to a power of $18 \mathrm{~W}$ coupled into the ACC, we used a high speed, low noise photodetector optimized for $5 \mathrm{~mA}$ photocurrent. It consisted of a $0.5 \mathrm{~mm}$ diameter InGaAs photodiode (OSI FCI-InGaAs-500) with a $750 \Omega$ transimpedance amplifier. The photodetector had a bandwidth of $150 \mathrm{MHz}$ and the electronic noise was at least $6.5 \mathrm{~dB}$ below the combined optical and electronics noise measured in the experiment.

A different photodetector was used at higher power levels. This detector was optimized for $50 \mathrm{~mA}$ photocurrent and consisted of a $1 \mathrm{~mm}$ InGaAs photodiode (Perkin Elmer C30641GH) with an $82 \Omega$ transimpedance amplifier. This photodetector had a bandwidth of $250 \mathrm{MHz}$ and the electronic noise was at least $6.2 \mathrm{~dB}$ below the total measured noise. The photodetector TFPD upstream of the ACC was used to calibrate the experiment.

We measured the relative power noise of the laser system with beam powers of $1,2,4,7,13,18,33$, and $67 \mathrm{~W}$ injected to the ACC. First we adjusted the impedance matching of the ACC using the micrometer screw such that the OACPD was not saturated. Then we measured the noise of the signals of the OACPD and TFPD using an Agilent 4395 A analyzer. To calibrate the OACPD signal as spectral density of the relative power noise upstream of the ACC, the bandwidth of the ACC and OMC, determined in different experiments, and the amplification $g$ of the optical ac coupling were necessary. The factor $g$ was critical for the calibration and depended on the impedance matching of the ACC and the parasitic power reflected by the ACC [3]. We determined $g$ for each measurement by matching the OACPD noise to the reference measurement performed with TFPD for frequencies between $300 \mathrm{kHz}$ and $1 \mathrm{MHz}$. At higher frequencies, the reference measurement was limited by electronic noise.

In a second step, we verified the calibration. We reduced the input power to about $2 \mathrm{~W}$ without touching the impedance matching, placed the EOAM into the beam path, and measured the transfer function from relative power modulation at the TFPD to relative power modulation at the OACPD. We fitted the model [2] to the measured transfer function in order to determine $g$.

In a different experiment, we measured the parasitic power at the OACPD in order to verify the suppression of the OMC for higher-order modes. In this experiment, we used a $2 \mathrm{~W}$ nonplanar ring oscillator (NPRO) [11] as a laser. We measured the amplification $g$ using the transfer function method mentioned above as a function of the power reflected by the ACC while adjusting its impedance matching. We determined the parasitic power by fitting the measurement to our model [3].

In our main experiment, we found a relative power noise of the laser system of about $10^{-7} \mathrm{~Hz}^{-1 / 2}$ at $300 \mathrm{kHz}$ steeply rolling off to $10^{-10} \mathrm{~Hz}^{-1 / 2}$ for frequencies above $10 \mathrm{MHz}$ (Fig. 2). At high frequencies, all measurements were limited by quantum noise at the expected level. Our detection system achieved a quantum-noise-limited peak sensitivity of $1.1 \times 10^{-10} \mathrm{~Hz}^{-1 / 2}$ at $27 \mathrm{MHz}$ at an input power of $67 \mathrm{~W}$ [Fig. 2(d)]. Thus, we were able to measure the technical power noise of the laser system down to a level of $10^{-10} \mathrm{~Hz}^{-1 / 2}$. The optical ac-coupled photodetector OACPD detected a photocurrent of $23 \mathrm{~mA}$. To reach the same sensitivity in a setup without optical ac coupling, an equivalent photocurrent of $32 \mathrm{~A}$ would have to be detected.

To demonstrate the high sensitivity-gain of the optical ac coupling technique, we performed one measurement without optical ac coupling at a photocurrent of $4.6 \mathrm{~mA}$ [Fig. 2(a)]. This measurement was limited by quantum noise at $8 \times 10^{-9} \mathrm{~Hz}^{-1 / 2}$ for frequencies above $5 \mathrm{MHz}$. 


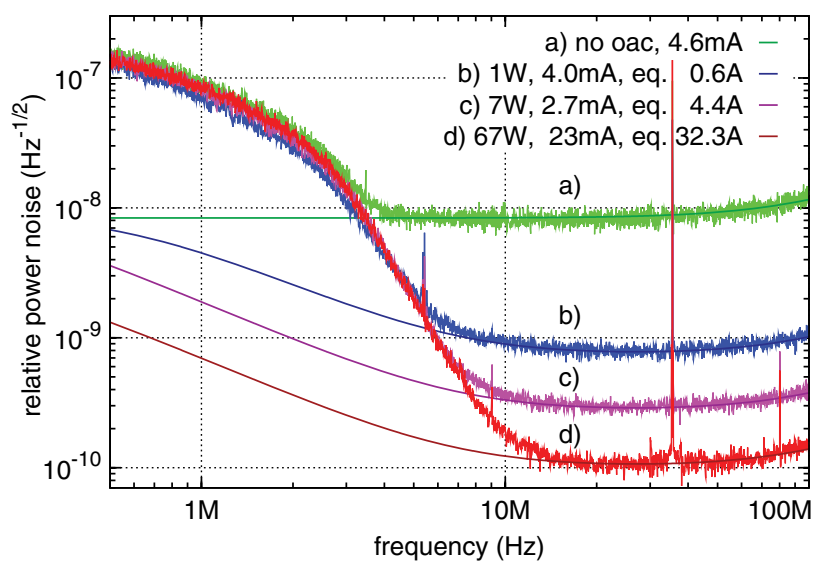

Fig. 2. (Color online) (b)-(d) Relative power noise of the laser system at different input powers to the experiment. The technical laser noise rolls off steeply toward higher frequencies. The measurements are limited by quantum noise (solid lines) at high frequencies at different levels. The input power, the average detected photo current, and the equivalent photo current are given in the legend. (a) For comparison, a measurement without optical ac coupling was performed demonstrating the high sensitivity gain of the optical ac coupling technique. The peak at $35.5 \mathrm{MHz}$ corresponds to a modulation frequency used in the laser internal control.

The parasitic power determination with the NPRO yielded a parasitic power at OACPD of 79 parts per million, allowing a maximum amplification of $g \approx 57$. The high power laser system had a better beam quality than the NPRO. Thus, less parasitic power was at the OACPD during the high power experiment and we were able to achieve in this setup a maximum amplification of $g=62.3$, which corresponds to a suppression of the carrier power by $g^{2}=3881$ in reflection of the ACC.

The total detection system had a responsiveness of 0.48 to $0.70 \mathrm{~A} / \mathrm{W}$ including the photodiode quantum efficiency, the throughput, alignment and mode-matching of the OMC, losses at the ACC, and several beam pick offs.

The experiment was limited to a maximal input power of $67 \mathrm{~W}$ due to thermal effects in the ACC and optical components between the ACC and OMC. These caused low frequency fluctuations in modematching and alignment such that we were not able to perform measurements at higher input powers.

In conclusion, we can state that our detection system reached what we believe to be a hitherto unattained power noise sensitivity at the $10^{-10} \mathrm{~Hz}^{-1 / 2}$ level using a high power laser system and the optical ac coupling technique. A peak power of $67 \mathrm{~W}$ was detected at an equivalent photocurrent of $32 \mathrm{~A}$. The measurement was quantum-noise-limited for frequencies above $10 \mathrm{MHz}$.
The feasibility and efficiency of an additional mode cleaner between the ACC and photodetector was demonstrated.

This detection scheme might improve the sensitivity of many high precision experiments, such as experiments on the entanglement of macroscopic bodies [12] or gravitational wave detectors. Gravitational wave detectors, for example, require sensitive photodetectors for the stabilization of the laser power circulating in their Michelson interferometer. In principle, the power recycling cavity [4] of these instruments could be used as an ACC. However, the beam reflected at the power recycling cavity consists mainly of modulation sidebands and higher-order TEM modes. This parasitic power would limit the optical ac coupling amplification to small factors. The topology presented in this Letter, however, would suppress parasitic power with the additional mode cleaner making the full sensitivity gain accessible and therefore allow a more sensitive power detection.

This research is supported by the German Volkswagen Stiftung and the Deutsche Forschungsgemeinschaft (DFG) as a part of the QUEST cluster of excellence. The authors would like to thank Christina Bogan, Jan Pöld, Oliver Puncken, and Lutz Winkelmann for their support in the operation of the laser system and Albrecht Rüdiger for his helpful comments during the preparation of this manuscript.

\section{References}

1. H.-A. Bachor and T. C. Ralph, A Guide to Experiments in Quantum Optics (Wiley-VCH, 2004).

2. P. Kwee, B. Willke, and K. Danzmann, Opt. Lett. 33, 1509 (2008).

3. P. Kwee, "Laser characterization and stabilization for precision interferometry," Ph.D. thesis (Universität Hannover, 2010).

4. P. R. Saulson, Fundamentals of Interferometric Gravitational Wave Detectors (World Scientific, 1994).

5. B. Willke, Laser Photonics Rev. 4, 780 (2010).

6. P. Kwee, B. Willke, and K. Danzmann, Appl. Phys. B 102, 515 (2011).

7. L. Winkelmann, O. Puncken, R. Kluzik, C. Veltkamp, P. Kwee, J. Poeld, C. Bogan, B. Willke, M. Frede, J. Neumann, P. Wessels, and D. Kracht, Appl. Phys. B 102, 529 (2011).

8. T. Hansch and B. Couillaud, Opt. Commun. 35, 441 (1980).

9. A. E. Siegman, Lasers (University Science, 1986), Chap. 11.3.

10. A. Rüdiger, R. Schilling, L. Schnupp, W. Winkler, H. Billing, and K. Maischberger, Opt. Acta 28, 641 (1981).

11. I. Freitag, A. Tünnermann, and H. Welling, Opt. Commun. 115, 511 (1995).

12. H. Müller-Ebhardt, H. Rehbein, R. Schnabel, K. Danzmann, and Y. Chen, Phys. Rev. Lett. 100, 013601 (2008). 